\title{
Erratum: Polyfunctionality of Lysozyme Destabilase from the Medicinal Leech
}

\author{
I. P. Baskova ${ }^{a, 1}$ and L. L. Zavalova ${ }^{b}$ \\ ${ }^{a}$ Faculty of Biology, Moscow State University, Vorob'evy gory, Moscow, 119992 Russia \\ ${ }^{b}$ Shemyakin-Ovchinnikov Institute of Bioorganic Chemistry, Russian Academy of Sciences \\ ul. Miklukho-Maklaya 16/10, Moscow, 117997 Russia \\ Received July 2, 2007; in final form, September 13, 2007
}

DOI: $10.1134 / \mathrm{S} 1068162009060156$

In the paper by I.P. Baskova and L.L. Zavalova, "Polyfunctionality of Destabilase Lysozyme from a Medicinal Leech," 2008, vol. 34, no. 3, pp. 337-343, the grant INTAS (no. 05 1000008-8147) was not mentioned. 